\title{
Pollenschutz auf Japanisch
}

\author{
Japaner tragen nicht nur aus Angst vor einer Erkältung einen Mund- \\ schutz, die Allergiekranken unter ihnen setzen die Operationsmasken \\ auch im Kampf gegen Pollen ein. Die Wirksamkeit dieser mechani- \\ schen Allergenabwehr wurde jetzt in einer Studie untersucht.
}

n Japan leiden 13\% der Bevölkerung unter einer Zedernpollenallergie. Eine Studie prüfte die Wirksamkeit einer mechanischen Abwehr der Zedernpollen durch Operationsmaske und Schutzbrille an zehn gesunden Probanden. Ermittelt wurde die Pollenkonzentration in der Nase und an den Konjunktiven mit und ohne Schutz. Dazu wurden zunächst Pollen aus Nase und Augen mit Kochsalzlösung entfernt.
Die Probanden setzten sich dann bei einem 30-minütigem Spaziergang den Pollen aus - einmal mit, einmal ohne Maske und Brille. Sie trugen dabei einen Pollenstaubsammler mit sich, um die aktuelle Pollenbelastung festzuhalten. Anschließend wurden Nase und Augen erneut ausgewaschen.

Die ermittelte Pollenkonzentration in der Nase und an den Konjunktiven wurde durch Maske und Schutzbrille nicht signifikant reduziert. Allerdings konnte die „Polleninvasionsrate“, definiert als Verhältnis von Pollenanzahl zur Pollenbelastung der Luft, durch die Barrieren verringert werden - im Nasenlumen im Vergleich zu ungeschützten Nasen sogar statistisch signifikant. Dem wirkte starker Wind entgegen: Bei höherer Windstärke war der Effekt des mechanischen Schutzes stark begrenzt.

Fazit: Operationsmasken und Schutzbrillen können Pollenallergiekranken nur wenig Erleichterung verschaffen.

Gotoh $\mathbf{M}$ et al. Inhibitory effects of facemasks and eyeglasses on invasion of pollen particles in the nose and eye: a clinical study. Rhinology 2005; 43: 266-70

\section{Wechsel vom Stall ins Büro}

\section{Bevor in Finnland Asthma als berufsbedingt anerkannt wird, verlangen die Versicherungsgesellschaften ein aufwändiges Prüf- verfahren: Die Betroffenen werden standardisiert untersucht und bei positivem Ergebnis in einem Zentralregister erfasst. Die Auswertung dieses Registers spiegelt die sich verändernde Arbeitswelt wider.}

\footnotetext{
A rbeitsmediziner aus Helsinki analysierten alle dem Finnish Register of Occupational Diseases gemeldeten Fälle von berufsbedingtem Asthma der Jahre 1986 bis 2002. Dabei zeigten sich ein Anstieg der Inzidenz berufsbedingter Asthmafälle von 1986 bis 1995, danach ein leichter Abfall und anschließend eine Stabilisierung in den ersten Jahren des aktuellen Jahrhunderts. Zu Beginn des Berichtszeitraums bis 1990 übte die Mehrheit der Betroffenen einen landwirtschaftlichen Beruf aus (59\%). Dieser Prozentsatz ging dann bis 2002 deutlich zurück auf $42 \%$. Im selben Zeitraum stieg die Zahl der berufsbedingten Asthmaerkrankungen bei Angestellten. Wichtigste Ursache für den Anstieg waren Asthmafälle in der Folge von Schimmelpilzsensibilisierungen - u. a. Aspergillus-, Cladosporium-, Penicillium- und Fusarium-Spezies -, hervorgerufen durch eine vermehrte Exposition in Gebäuden mit Wasserschäden. Machten diese Fälle zwi-
}

schen 1986 und 1990 lediglich 0,5\% aller Meldungen aus, so waren es 2002 schon $18 \%$. Chemikalien, hauptsächlich Diisocyanate, Abgase beim Schweißen, Friseurchemikalien und Formaldehyd, wurden bei 10 bis 30\% der Fälle als Auslöser ermittelt, dieser Anteil fiel seit 1990.

Erklärt werden die beschriebenen Veränderungen durch den EU-Beitritt Finnlands 1995 und den damit verbundenen Rückgang der landwirtschaftlichen Betriebe. Der weltweit wohl einmalig hohe Anteil von Kuhepithelienallergien in Finnland wird auf die Tatsache zurückgeführt, dass die Tiere wegen des langen Winters überwiegend in Ställen gehalten werden und die Bauern dadurch intensiven Allergenkontakthaben. Klimabedingt ist auch die Zunahme der Schimmelpilzallergien bei Angestellten zu erklären: Die Bauweise vieler nach dem Zweiten Weltkrieg errichteter Bürogebäude wurde nicht den speziellen Witte-

$\begin{array}{lr}\text { Tabelle. Berufe von } & \mathbf{4} 8 \text { Patienten } \\ \text { mit berufsbedingtem } & \text { Asthma auf- } \\ \text { grund einer Schimmelpilzallergie } & \\ & \text { Anteil (\%) } \\ \text { Beruf } & 12 \\ \text { Lehrer } & 10 \\ \text { Krankenschwester } & 8 \\ \text { Büroangestellter } & 8 \\ \text { Kindergärtnerin } & 8 \\ \text { Andere soziale Berufe } & 4 \\ \text { Landwirt } & \\ \text { Angestellter im Nahrungsmittel } & 4 \\ \text { produzierenden Gewerbe } & 46 \\ \text { Sonstige } & \end{array}$

rungsanforderungen in Finnland angepasst, was sich in früher Baufälligkeit, Wasserschäden und dem damit verbundenen Schimmelpilzbefall niederschlägt.

Fazit: In Finnland sind die Fälle von berufsbedingtem Asthma in der Landwirtschaft zurückgegangen. Gleichzeitig stiegen die Schimmelpilz-bedingten Asthmaerkankungen bei Angestellten, die in Büros mit Feuchtigkeitsschäden arbeiten, dramatisch an.

Piipari R et al. Agents causing occupational asthma in Finland in 1986-2002: Cow epithelium bypassed by moulds from moisture-damaged buildings. Clin Exp Allergy 2005; 35: 1632-7 